\title{
Projeto Terapêutico Singular no manejo de casos complexos: relato de experiência no PET-Saúde Interprofissionalidade
}

\section{Singular Therapeutic Project in the management of complex cases: experience report in PEW-Health Interprofessionality}

\author{
Maynara Guaripuna Ferreira' ${ }^{10} \mid$ mayguaripuna@gmail.com \\ Kathleen Hanna Gonçalves Ferreira' (D) kathleenhanna73@gmail.com \\ Cibelle Regina Piani de Souza' (D) cibelle.piani@gmail.com \\ Priscila Cherley de Araújo Ortiz² (i) priscila.nutricionista@yahoo.com.br \\ Rodrigo Guimarães dos Santos Almeida' (D) rgclaretiano@gmail.com \\ Adélia Delfina da Motta Silva' (1D) adelia.motta.silva@gmail.com
}

\begin{abstract}
RESUMO
Introdução: O Programa de Educação pelo Trabalho para a Saúde (PET-Saúde) surgiu em 2008, numa parceria entre o Ministério da Saúde e o Ministério da Educação, com o propósito de fomentar a integração ensino-serviço-gestão-comunidade e oportunizar vivências para profissionais, estudantes, professores e usuários dos serviços, em consonância com as necessidades do SUS. A nona edição (2019-2021) do programa teve como tema a interprofissionalidade. Este relato objetiva compartilhar a aprendizagem interprofissional a partir da experiência de um Projeto Terapêutico Singular (PTS) para um caso complexo dentro das atividades do PET-Saúde de uma universidade da Região Centro-Oeste, realizado em uma unidade de saúde da família (USF), em Campo Grande, em Mato Grosso do Sul (MS).

Relato de experiência: Participaram desta experiência acadêmicas de um grupo tutorial do PET-Saúde Interprofissionalidade, preceptoras, profissionais do Núcleo Ampliado de Saúde da Família e Atenção Primária (Nasf-AP), e tutora, docente de uma Faculdade de Medicina. O PTS foi desenvolvido com paciente idosa, com polifarmácia, com as seguintes condições crônicas: diabetes mellitus (DM), depressão (DEP) e hipertensão arterial sistêmica (HAS). A paciente foi acompanhada durante o período de agosto de 2019 a fevereiro de 2020, anterior à pandemia da Covid-19, em uma USF, em Campo Grande-MS, selecionada pela equipe em virtude da complexidade do caso.

Discussão: Por meio do PTS, o grupo teve a oportunidade de avaliar, auxiliar e executar práticas para fortalecer o "projeto de felicidade" da paciente. O PTS permite a intersubjetividade entre equipe e paciente, centrando-se não apenas nas doenças, mas também no indivíduo. As visitas domiciliares realizadas proporcionaram momentos de escuta para a condução do cuidado em conformidade às necessidades sentidas e não sentidas pela usuária. Na prática do PTS, presta-se cuidado integral, focado no indivíduo, de modo a apresentar as potencialidades na educação permanente e no trabalho em equipe interprofissional, compartilhar saberes, aprimorar a sustentabilidade do cuidado e, consequentemente, qualificar a assistência em saúde, corroborando os resultados obtidos nesta experiência.

Conclusão: A participação no programa permitiu vivências antes ausentes na graduação, como o contato com os diferentes cursos da saúde, a prática nos cenários reais do SUS e a aplicação dos conceitos vistos apenas na teoria, como o atendimento humanizado e olhar integral, além da comunicação com a equipe e paciente.
\end{abstract}

Palavras-chave: Atenção Primária à Saúde; Diabetes Mellitus; Pé Diabético; Hipertensão; Assistência Centrada no Paciente.

\section{ABSTRACT}

Introduction: The Program Education through Work for Health (PEW-Health) was created in 2008 as a partnership between the Ministries of Health and Education, promoting the teaching-service-management-community integration, providing opportunities for professionals, students, teachers, and service users, in line with the needs of SUS, and having the topic of interprofessionality for its ninth edition (2019-2021). This report aims to share interprofessional learning based on the experience of a singular therapeutic project (STP) for a complex case within the PEW-Health activities of a university in the Midwest region, carried out in a Family Health Unit (FHU) in the municipality of Campo Grande, Mato Grosso do Sul (MS).

Experience report: Students from a tutorial group of PEW-Health Interprofessionality participated in this experience, as well as preceptors, professionals from the Extended Nucleus of Family Health and Primary Care (ENFH-PC), and a tutor, who was a teacher in a Medical School. The STP was developed with an elderly patient with polypharmacy and the following chronic conditions: diabetes mellitus (DM), depression (DEP) and systemic arterial hypertension (SAH), monitored during the period from August 2019 to February 2020, prior to the COVID-19 pandemic, in a FHU in Campo Grande-MS, selected by the team due to the complexity of the case.

Discussion: Through the STP, the group had the opportunity to evaluate, assist and perform practices to strengthen the patient's "happiness project". The STP allows team-patient intersubjectivity, focusing not only on the diseases, but on who they are. The carried-out home visits provided moments of listening for the performance of care in accordance with the needs, perceived and not perceived by the user. In the practice of STP, comprehensive care is provided, focused on the individual, showing the potential in continuing education and interprofessional teamwork, sharing knowledge, improving the sustainability of care and, consequently, qualifying health care, corroborating the results obtained in this study.

Conclusion: Participation in the program allowed experiences that were previously absent during undergraduate school, such as contact with different health courses, practice in real SUS scenarios and application of concepts seen only in theory, such as humanized care and a comprehensive view, as well as communication with the team and the patient.

Keywords: Primary Health Care; Diabetes Mellitus; Diabetic foot; Hypertension; Patient-Centered Care.

\footnotetext{
${ }^{1}$ Universidade Federal de Mato Grosso do Sul, Campo Grande, Mato Grosso do Sul, Brasil.

${ }^{2}$ Secretaria Municipal de Saúde, Campo Grande, Mato Grosso do Sul, Brasil.

Editora-chefe: Rosiane Viana Zuza Diniz. | Editora associada: Ana Cláudia Santos Chazan.

Recebido em 21/09/21; Aceito em 07/12/21. | Avaliado pelo processo de double blind review.
} 


\section{INTRODUÇÃO}

Para consolidar os princípios doutrinários e organizativos do Sistema Único de Saúde (SUS), vários movimentos vêm sendo realizados pelos Ministérios da Saúde e da Educação, ao longo dos anos, com o intuito de integrar o cuidado, a partir da intersetorialidade das profissões desde a graduação, de modo a induzir a formação de profissionais aptos ao trabalho em equipe e a produção colaborativa, e promover a interprofissionalidade, sempre em consonância com as Diretrizes Curriculares Nacionais (DCN) para cursos da área da saúde ${ }^{1-5}$. Contudo, a vivência da interprofissionalidade durante a graduação ainda é um desafio, considerando a incompatibilidade das grades curriculares dos cursos da saúde, os diversos cenários de prática e as questões concernentes à integração ensino-serviço-gestão-comunidade no SUS ${ }^{2,6}$.

Nessa perspectiva, o Programa deEducação peloTrabalho para a Saúde (PET-Saúde) surgiu em 2008, numa parceria entre o Ministério da Saúde e o Ministério da Educação, a fim de fomentar a integração ensino-serviço-gestão-comunidade e oportunizar vivências para profissionais, estudantes, professores e usuários dos serviços, em consonância com as necessidades do SUS ${ }^{7}$. A nona edição (2019-2021) do programa teve como tema a interprofissionalidade, apontando para o plano de ação definido para implementação de mudanças nas práticas assistenciais, conforme estabelecido pela Organização Pan-Americana da Saúde ${ }^{3,7}$.

A educação interprofissional (EIP) acontece quando "estudantes de duas ou mais profissões aprendem sobre os outros, com os outros e entre si para possibilitar a efetiva colaboração e melhorar os resultados na saúde" (p. 10) Esse formato procura integrar ensino-serviço por meio da participação de trabalhadores do SUS, docentes e acadêmicos da área da saúde, dos diferentes cursos, que juntos desenvolvem atividades voltadas ao cuidado integral, em qualquer nível da atenção à saúde ${ }^{2,3,5}$.

A EIP tem como premissa a transformação das práticas em saúde, por meio da promoção da colaboração entre profissionais e do aprendizado do efetivo trabalho em equipe, preconizando o aprender juntos para trabalhar juntos. Portanto, adotar os pressupostos da EIP é uma aposta para mudar práticas de saúde e gerar trabalho interprofissional no contexto do SUS.

Enquanto a interprofissionalidade postula a interação colaborativa com respeito ao conhecimento específico de cada área, a fim de melhorar a qualidade dos serviços e o caráter do cuidado $^{10}$, a multiprofissionalidade corrobora a fragmentação do cuidado e a balização dos saberes ${ }^{11}$. Entende-se, portanto, que a interprofissionalidade converte a produção do cuidado específico em cuidado integral, promovendo trabalho em equipe, integrando diferentes núcleos profissionais, de forma colaborativa, com foco nas necessidades de saúde de usuários e população ${ }^{11}$.

No âmbito da interprofissionalidade, o Projeto Terapêutico Singular (PTS), dispositivo da Política Nacional de Humanização (PNH), surge como potente ferramenta de aprendizagem e cuidado na atenção primária à saúde (APS) que considera a historicidade e as necessidades do paciente. Dessa forma, trata-se da transformação de um instrumento de gestão do cuidado em um modelo de assistência integral que visa à produção de autonomia, protagonismo e inclusão social do sujeito ${ }^{12-15}$.

O PTS apresenta-se como instrumento de organização do cuidado, construído entre equipe e usuário, considerando as singularidades do sujeito e sua complexidade. Casos complexos, frequentes na $\mathrm{APS}^{16}$, exigem soluções complexas e revelam a necessidade de considerar questões transversais na produção de cuidado, compreendendo um conjunto de propostas de condutas terapêuticas que exige articulação entre diferentes profissionais e utilização das reuniões de equipe para refletir sobre o caso e possíveis soluções ${ }^{12,13,17}$. Assim, ao exigir tal articulação e trabalho em equipe, colaborativamente, o PTS aproxima-se da EIP, possibilitando a efetiva colaboração entre profissionais e a melhoria nos resultados do cuidado integral em saúde ${ }^{11}$.

Assim, este artigo tem como objetivo relatar a aprendizagem interprofissional a partir da experiência de estruturação de um PTS para um caso complexo dentro das atividades de extensão do PET-Saúde da Universidade Federal de Mato Grosso do Sul (UFMS) realizado em uma unidade de saúde da família (USF), no município de Campo Grande, em Mato Grosso do Sul (MS).

\section{RELATO DE EXPERIÊNCIA}

Participaram desta experiência seis acadêmicas integrantes de um grupo tutorial do PET-Saúde Interprofissionalidade, dos seguintes cursos da área da saúde: Enfermagem, Farmácia, Fisioterapia, Medicina, Odontologia e Psicologia - entre o segundo e o terceiro ano da graduação, do sexo feminino, entre 19 e 22 anos; duas preceptoras profissionais da APS da área da nutrição e fisioterapia integrantes do Núcleo Ampliado de Saúde da Família e Atenção Primária (Nasf-AP); e uma tutora, docente da Faculdade de Medicina da UFMS, cirurgiã-dentista. O PTS foi desenvolvido com uma paciente idosa, branca, em uso de polifármacos, com as seguintes condições crônicas: diabetes mellitus (DM), depressão (DEP) e hipertensão arterial sistêmica (HAS), acompanhada durante o período de agosto de 2019 a fevereiro de 2020, anterior à pandemia da coronavirus disease 2019 (Covid-19), em uma USF de Campo Grande, selecionada pela equipe em virtude da complexidade do caso. 
O relato desta experiência foi autorizado mediante assinatura de Termo de Consentimento Livre e Esclarecido (TCLE), estando vinculado ao projeto "Interprofissionalidade na percepção de discentes, docentes, profissionais e usuários do Sistema Único de Saúde", aprovado pelo Parecer nº 3.780.149 - Certificado de Apresentação para Apreciação Ética (CAAE) no 2 22845619.1.0000.0021 - da Comissão de Ética e Pesquisa em Seres Humanos da UFMS.

O PTS foi constituído dos seguintes movimentos e momentos de cuidado: escolha do caso, visita domiciliar, diagnóstico situacional, formulação de hipóteses para intervenção, discussão dos objetivos entre a equipe, definição de metas de curto, médio e longo prazos, e, posteriormente, reavaliação do caso e resultados.

O caso foi escolhido a partir da temática condições crônicas, e, nas visitas domiciliares, avaliaram-se as condições de vida, moradia, higiene, saúde, convívio social, histórico, relacionamentos familiares e morbidades. As atividades foram diretamente acompanhadas pela tutora e pelas preceptoras. A usuária apresentava dificuldades de adesão ao tratamento e conflitos de vínculo com a USF, além das condições crônicas coexistentes. No primeiro encontro, as estudantes, as preceptoras e a tutora foram apresentadas, e explicou-se o motivo da visita. Já no primeiro momento, houve uma aproximação com a paciente e obteve-se um maior conhecimento acerca do histórico dela no SUS. Natural de São Paulo, a paciente, que é aposentada, vive sozinha em Campo Grande, tem 68 anos e é divorciada. Outras características da paciente: DM tipo II, HAS, DEP, com polifarmácia, não tabagista, não alcoolista e alfabetizada. Teve três gestações, dois partos e um aborto, possui duas filhas que moram em outro estado, com as quais apresenta relacionamento estreito e conflituoso. Relatou relacionamento próximo com a irmã que reside em uma cidade do interior. Também apresenta histórico de cirurgia no joelho esquerdo.

Seguindo as etapas do PTS, o diagnóstico teve início com avaliação e problematização das singularidades, das necessidades, dos fatores sociais, econômicos e biológicos, dos desejos da paciente, entre outros. As visitas domiciliares aconteceram semanalmente, mostrando-se fundamentais para estabelecimento do vínculo. A cada contato, a paciente relatava os assuntos com maior profundidade e demonstrava confiança na equipe ${ }^{18}$.

Utilizaram-se ferramentas de abordagem familiar, genograma e ecomapa, para propor estratégias a serem executadas no cuidado (Figuras 1 e 2). Enquanto o genograma produz um desenho da estrutura familiar, representando parentescos, idades dos membros, doenças, fatores de risco e a complexidade das relações familiares, o ecomapa permite a avaliação familiar, complementar ao genograma, que inclui outros laços afetivos associados ao indivíduo, conectando pessoas e famílias às redes sociais de apoio, necessárias à produção de cuidado ${ }^{13,19,20}$.

Taisinstrumentosforam construídos com esboços manuais conforme as informações eram relatadas. Posteriormente, o genograma foi desenhado com a ferramenta "Álbum de Família" do Núcleo de Educação em Saúde Coletiva (Nescon) da Universidade Federal de Minas Gerais (UFMG) ${ }^{19,21}$. Para o ecomapa, utilizou-se a plataforma de design gráfico Canva ${ }^{22}$.

Ao utilizar tais ferramentas, o grupo observou a repetição de padrões de adoecimento, o núcleo familiar da paciente, tendo melhor percepção do contexto social. Elas ajudaram a estabelecer metas pertinentes e identificar possíveis suportes sociais ${ }^{20,23}$. O ecomapa evidenciou vulnerabilidades da paciente, visto que pessoas com quem apresentava relações mais fortes eram fisicamente distantes, à exceção da vizinha $M$, com a qual possuía vínculo de maior destaque, contudo também conflituoso.

Realizou-se também avaliação do pé diabético, seguindo as recomendações do Caderno de Atenção Básica Estratégias para o cuidado da pessoa com doença crônica: diabetes mellitus ${ }^{24}$, com inspeção da pele, das unhas e dos dedos; observação à procura de deformidades ósseas; aferição do pulso tibial posterior e pedioso; teste de sensibilidade com monofilamento e diapasão (sensibilidade vibratória) e do reflexo aquileu. Detectaram-se micoses interdigitais, dedos em garra, alterações de coloração e temperatura da pele, perda de sensibilidade, rachaduras e formigamento, classificando-se como grau 2 quanto à categoria de risco ${ }^{18}$, que corresponde à doença arterial periférica (DAP) com ou sem perda da sensibilidade protetora (PSP).

A definição de metas de curto, médio e longo prazos negociadas com a usuária respeitou os princípios propostos para o PTS ${ }^{13,25}$. A primeira meta foi a melhora da condição do pé diabético por meio de ações como corte adequado das unhas, hidratação da pele, orientações sobre os calçados adequados e cinesioterapia, visando à melhora da vascularização periférica ${ }^{26-28}$.

Também foi necessário abordar a questão da polifarmácia que, de acordo com Mangin et al. ${ }^{29}$, pode representar riscos reais para a boa saúde de idosos e, sobretudo, daqueles com comorbidades. Destaca-se que regimes complexos de medicamentos costumam ultrapassar a capacidade de manejo do paciente, reduzindo a adesão, especialmente nas pessoas mais velhas, o que verificamos na prática com a paciente que não era capaz de organizar sozinha de forma adequada a ingesta dos diferentes medicamentos prescritos. Embora o grupo tenha tido dificuldades em alterar a prescrição, pois não 
Figura 1. Genograma do caso relatado, construído a partir da ferramenta "Álbum de Família" do Núcleo de Educação em Saúde Coletiva (Nescon) da UFMG - Campo Grande/MS, 2020.

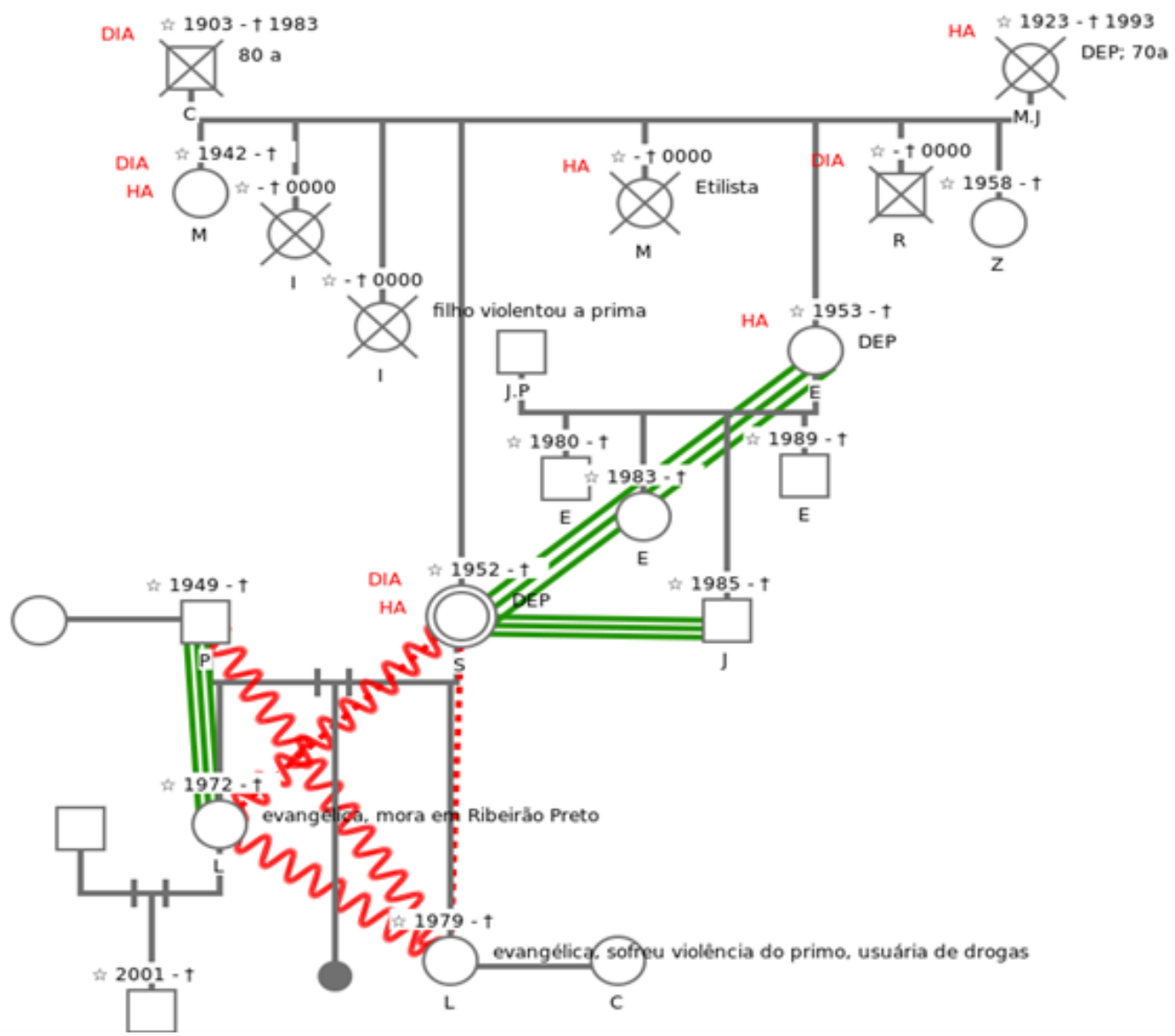

$\mathrm{A}=$ anos; $\mathrm{DEP}=$ depressão; $\mathrm{DIA}=$ diabetes mellitus; $\mathrm{HA}=$ hipertensão arterial sistêmica. Siglas geradas automaticamente pelo sistema . Fonte: Elaborada pelos autores.

Figura 2. Ecomapa construído, a partir do aplicativo Canva, em conjunto com a paciente - Campo Grande/MS, 2020.

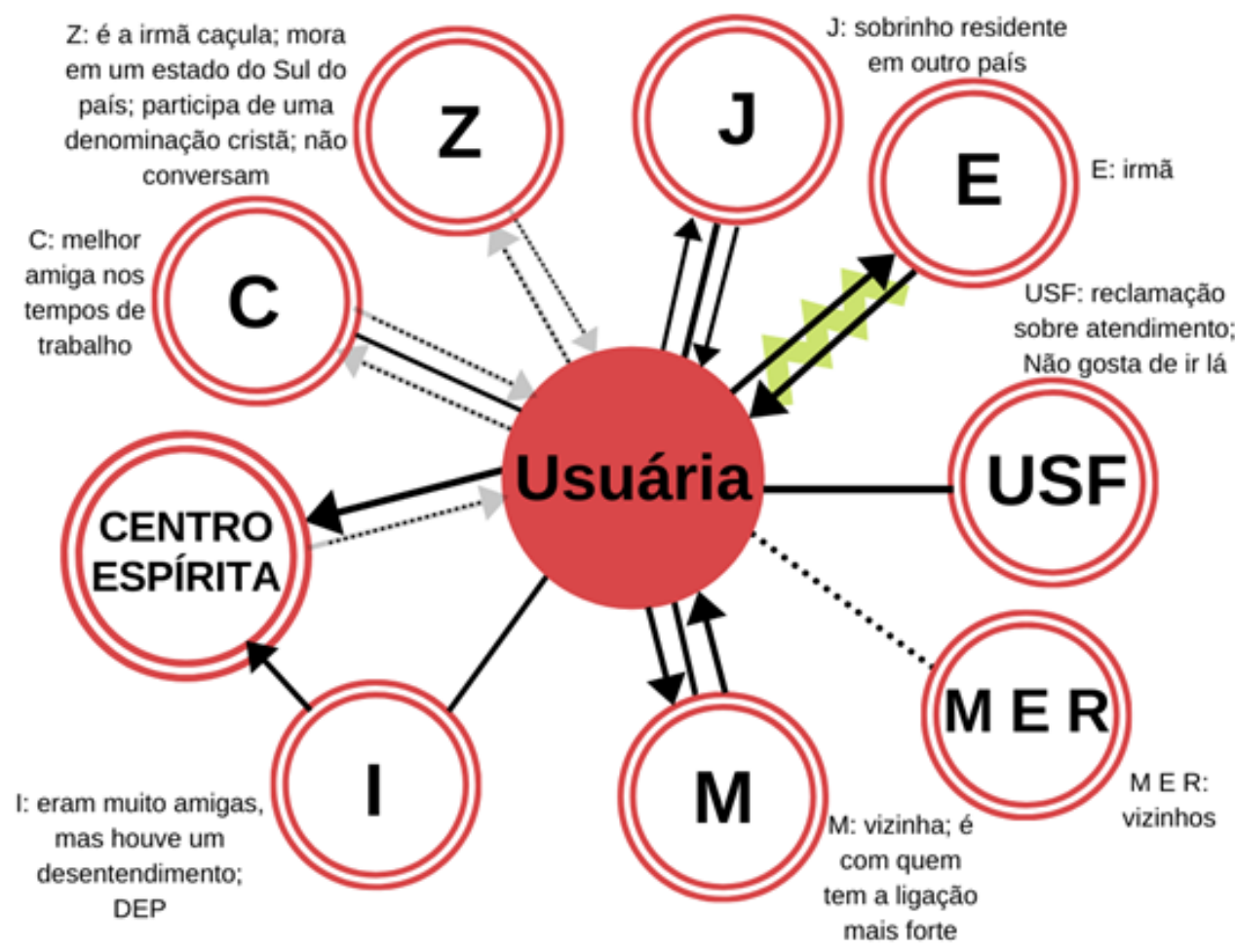

DEP = depressão; USF = unidade de saúde da família.

Fonte: Elaborada pelos próprios autores. 
Figura 3. Primeiros cuidados para o uso racional dos medicamentos: revisão da receita e reorganização da caixa de medicamentos da paciente, com a retirada dos exemplares vencidos - Campo Grande/MS, 2020.

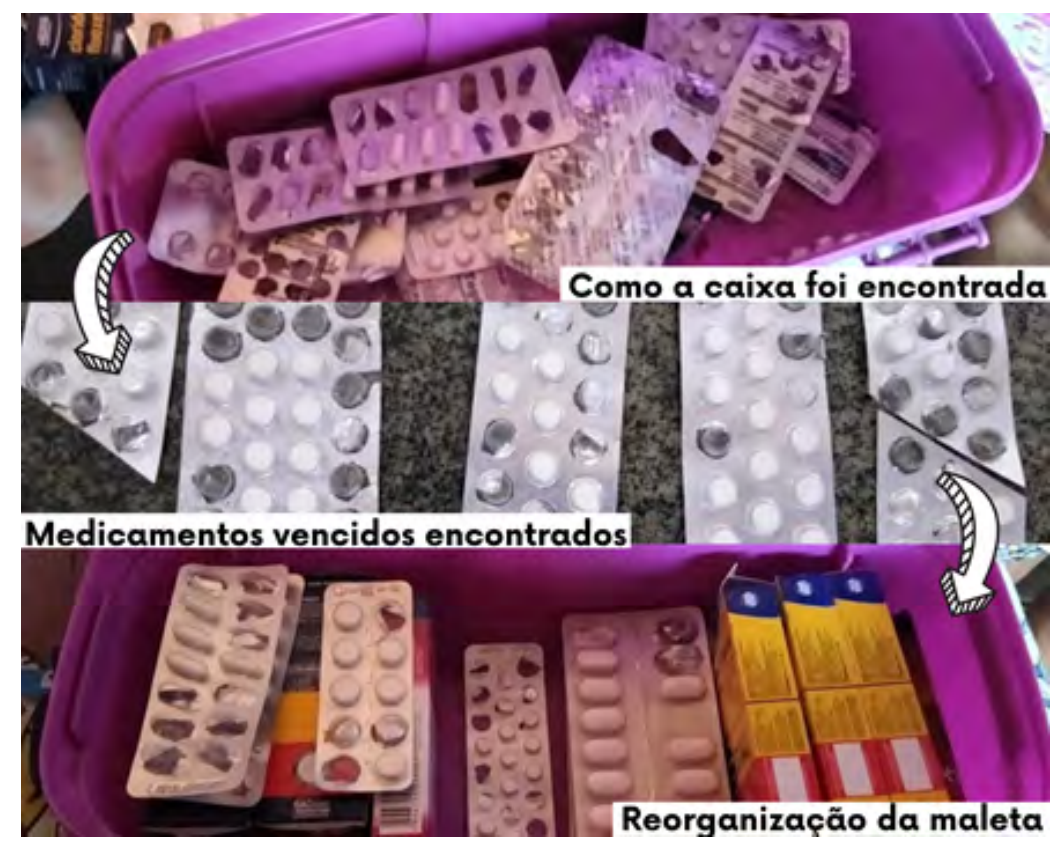

Fonte: Elaborada pelos autores.

havia médico na equipe responsável pela usuária, buscou-se, ao reorganizar a caixa de medicamentos (Figuras 3 e 4) e criar formulário para marcação das ingestas e alarmes nos horários, garantir o uso racional dos medicamentos, assim como, olhando para questões como solidão e isolamento social, dar voz às necessidades sentidas pela paciente, ações tão importantes quanto a prescrição ${ }^{29}$.

A intervenção na prescrição aconteceu pelos relatos de inadequação do uso, dificuldade para compreender a prescrição e medicamentos com prazo ultrapassado. Num primeiro momento, foi feita a reorganização da "maleta" de medicamentos da paciente (Figura 3). Outra estratégia foi contatar a USF para agenda de consulta com o único médico disponível na unidade, de outra equipe, visando à atualização das receitas. Posteriormente, providenciou-se uma tabela com os nomes dos remédios e os horários estipulados. Ademais, as acadêmicas confeccionaram uma caixa com divisórias (Figura 4) para que a paciente guardasse os medicamentos corretamente, separando-os com as mesmas cores que estavam presentes na tabela, além de propiciar o descarte correto das medicações vencidas. Considerando os diferentes horários de ingesta das medicações, as acadêmicas programaram o celular da paciente para lembretes (alarmes) nesses horários.

Na lógica de construção de projetos de felicidade ${ }^{30,31}$, resgataram-se sonhos de ressocialização, pois a paciente compartilhou com o grupo que seu grande desejo era retornar à universidade, já que não havia conseguido completar a graduação por dificuldade de pagamento. Uma oportunidade
Figura 4. Após busca de alternativas, foi confeccionada nova caixa de medicamentos pelas acadêmicas - Campo Grande/MS, 2020.

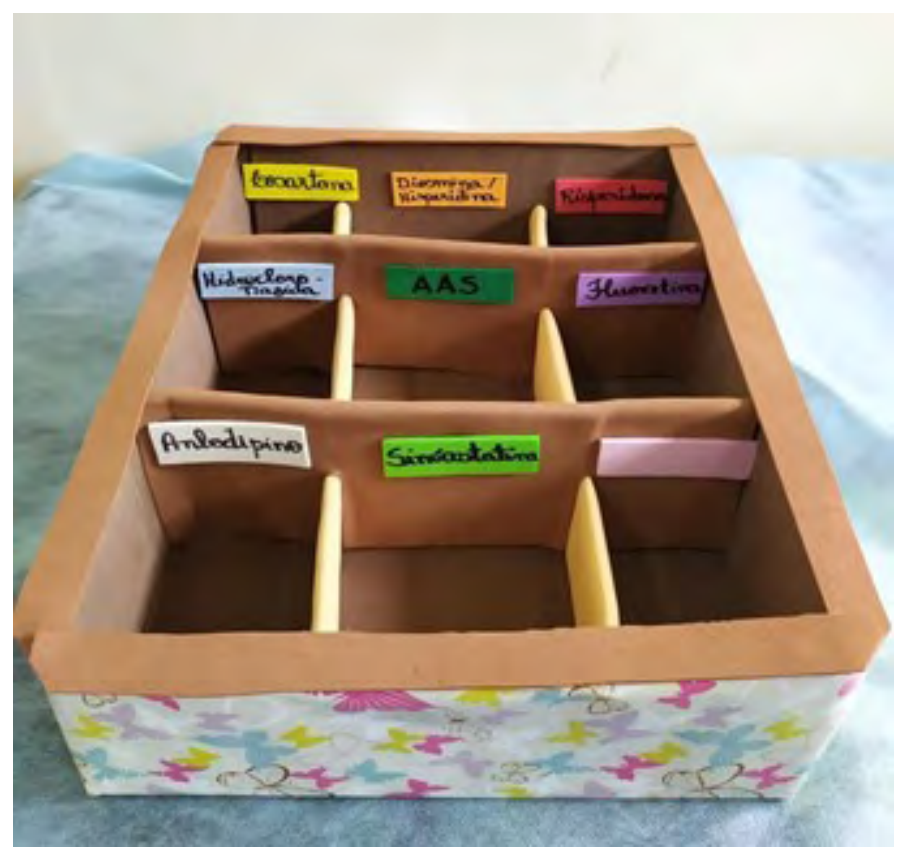

Fonte: Elaborada pelos autores.

de ressocialização inicial foi o incentivo à participação nos grupos de atividades físicas promovidos pelo Nasf-AP na USF, permitindo o restabelecimento de vínculo com a unidade. Em médio prazo, buscou-se a inserção no Programa Institucional da Universidade Aberta à Pessoa Idosa (UnAPI/UFMS), com 
participação semanal da oficina de memória "MemorIDADE", em que são desenvolvidas atividades de estimulação cognitiva e psicossocial, firmadas no conceito da aprendizagem ao longo da vida ${ }^{32}$.

Ademais, foram desenvolvidas ações de educação em saúde personalizadas. Cabe destacar a dinâmica para a compreensão das condições crônicas e consequências no organismo. Para isso, utilizou-se o Mapa de Conversação "Como o Corpo e a Diabetes Funcionam"33, uma estratégia educativa com ilustrações interativas sobre situações cotidianas da pessoa que convive com $\mathrm{DM}^{34}$. Outros temas trabalhados incluíram alimentação saudável, cuidados com o pé diabético e saúde da mulher.

A cada etapa do processo, a ficha do PTS foi atualizada com os procedimentos e as informações. A divisão de responsabilidades ocorreu nas reuniões e discussões da equipe sobre o caso, bem como nas visitas domiciliares, sempre respeitando o protagonismo da paciente e sua capacidade de assumir mudanças. Outrossim, seguindo o processo de reavaliação, os procedimentos realizados na etapa do diagnóstico foram reaplicados. Por meio de reexames, conversas, atitudes e relatos, tanto da usuária quanto de membros da equipe da USF, notou-se a evolução do caso, principalmente em relação ao pé diabético (Figura 5).

Vale ressaltar que as ferramentas utilizadas na APS fomentaram a troca de saberes entre as diversas formações dos acadêmicos e profissionais, que compreenderam a diferença entre as práticas comuns, específicas e colaborativas, e, assim, reconheceram a possibilidade de troca de saberes a partir de dispositivos como o PTS. A avaliação dessa prática ocorreu de forma coletiva e processual pelos participantes, tendo a tutora o papel de disparadora desse processo que, ao final, foi compartilhado com a usuária, sendo verbalizada sua percepção.

\section{DISCUSSÃO}

Em contraste com os modelos convencionais de cuidados, que primam pela assistência curativa, especializada, fragmentada e individual, a proposta de trabalho do Nasf-AP é a de superação dessa lógica em direção à corresponsabilização e à gestão integrada do cuidado por meio de atendimentos compartilhados e projetos terapêuticos que envolvam os usuários, na APS, sendo capazes de considerar a singularidade dos sujeitos ${ }^{35}$, o que foi vivenciado na prática pelo grupo. Diante da proposta metodológica do núcleo para o trabalho interdisciplinar e interprofissional, é necessária a revisão crítica dos processos educativos e formativos que vêm sendo disponibilizados por instituições de ensino superior na formação de profissionais da área da saúde ${ }^{13,35}$, pois o trabalho interprofissional, de fato, não tem sido proporcionado na graduação como rotina - nem na prática, nem nos projetos pedagógicos dos cursos $(P P C)^{11}$, sendo o PET-Saúde uma oportunidade, ainda que no formato de extensão, para tal aprendizagem.

Assim, destaca-se que, por meio do PTS, o grupo teve a oportunidade de avaliar, auxiliar e executar práticas, com vistas a fortalecer o "projeto de felicidade" ${ }^{\prime 30,31}$ da paciente, desenvolvendo competências comuns e complementares, bem como as dimensões colaborativas da EIP $^{36}$, e aprendendo

Figura 5. Evolução do cuidado do pé diabético - Campo Grande/MS, 2020.
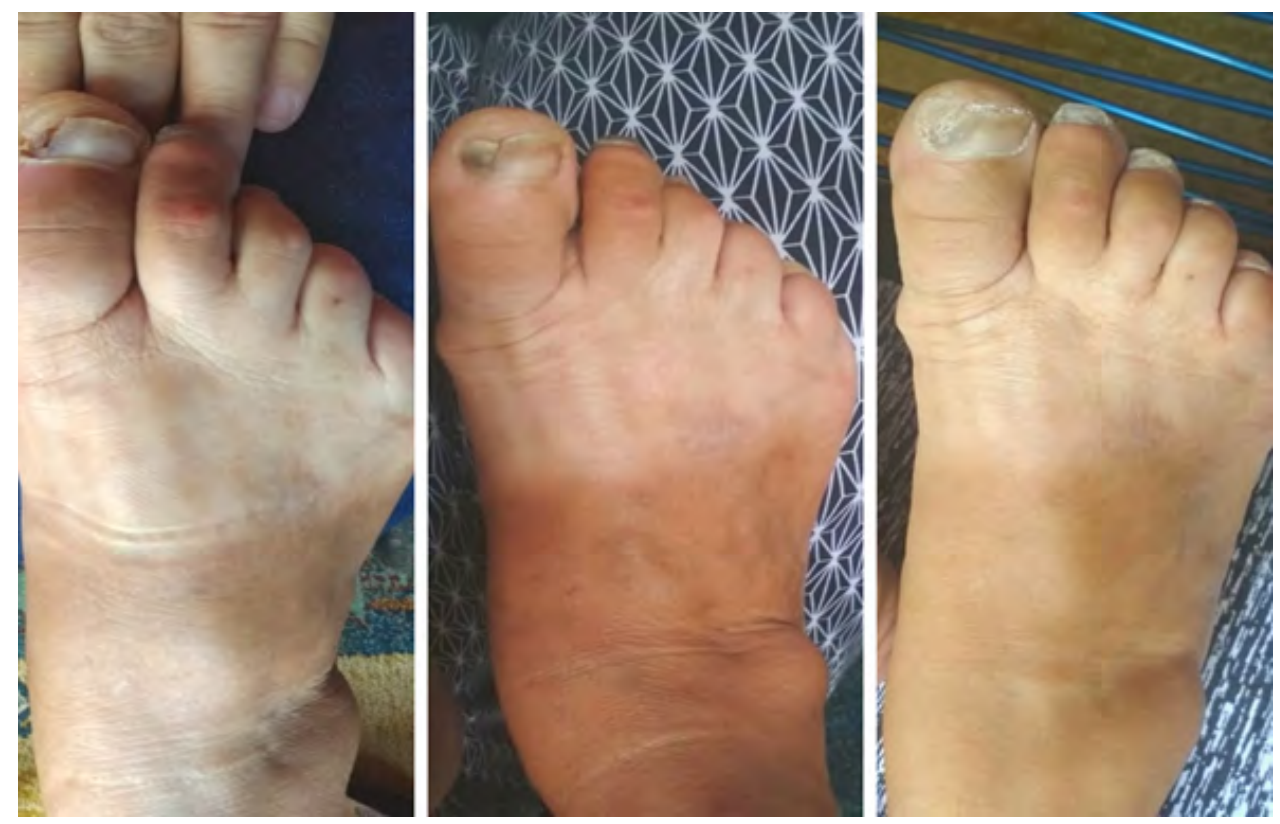

Fonte: Elaborada pelos autores. 
sobre o cuidado integral destinado a pessoas com condições crônicas ${ }^{6,10,27}$. O PTS permite a intersubjetividade entre equipe e paciente, centrando-se não apenas nas doenças, mas também no indivíduo. A felicidade pode ser compreendida com um valor estruturado a partir de vivências reais e necessárias para se viver, conscientizando o indivíduo a reconhecer suas próprias experiências e trocas estabelecidas com outros indivíduos, sendo importante que os agentes promotores das práticas em saúde estejam mais atentos e flexíveis aos anseios do usuário, ouvindo e compartilhando experiências e propósitos, para estabelecer um vínculo por meio do diálogo ${ }^{31}$.

Durante o semestre, o grupo foi desafiado a pensar na paciente e cuidar dela, escutando suas demandas, reconhecendo seu "projeto de felicidade, isto é, que concepção de vida bem-sucedida" ${ }^{\prime 37}$ (p. 68) orientava a vida daquela mulher que estava sendo cuidada. Nesse processo, pode-se afirmar que o grupo teve a oportunidade de aprender sobre o cuidar de forma humanizada ao abrir espaço para o diálogo em todos os encontros terapêuticos, assumir e compartilhar responsabilidades, reconstruir identidades ${ }^{30,31}$.

A partir da realização desse PTS, com o tempo e por meio da comunicação efetiva e afetiva com o grupo, a paciente compartilhou angústias e ambições, histórias, suas marcas físicas e psicológicas, o que possibilitou uma intervenção mais precisa da equipe. As visitas domiciliares realizadas proporcionaram momentos de atenção e escuta para a condução do cuidado em conformidade às necessidades sentidas e não sentidas pela usuária ${ }^{38}$.

O fundamento e a importância do cuidado nas ações de saúde se dão pela promoção de atitudes que induzam encontros intersubjetivos, auxiliados por tecnologias, porém não restritas a elas, visando à humanização do cuidado ${ }^{37}$. Macedo et al. ${ }^{39}$ corroboram este relato ao concluírem que existem configurações extrínsecas e intrínsecas que englobam o ser humano, as quais envolvem aspectos sociais, culturais e pessoais, e, por isso, é necessário detectar os mais pertinentes de cada circunstância, importantes orientadores para a intervenção necessária, tendo consciência de que se trata de um processo dinâmico que requer cuidados diferenciados a cada momento. Por sua vez, Mendes et al. ${ }^{40}$ relatam a eficiência na prática do PTS, no que concerne ao cuidado integral focado no indivíduo. O PTS, segundo esses autores, apresenta potencialidades na educação permanente e no trabalho em equipe interprofissional, ao compartilhar saberes entre os integrantes, aprimorar a sustentabilidade do cuidado e, consequentemente, qualificar a assistência em saúde, o que corrobora os resultados obtidos neste relato.

A experiência aqui relatada reforça as orientações dadas pelo Conselho Nacional de Saúde em sua Resolução n 569, de 8 de dezembro de $2017^{2}$, que apontam para o trabalho interprofissional como orientador das DCN de cursos da área da saúde, a fim de que o trabalho em equipe se efetive na prática, promovendo a integralidade tanto dos diferentes núcleos de saberes profissionais quanto do cuidado e, ainda, a intersetorialidade, integrando diferentes setores da sociedade para solucionar situações complexas de necessidades de saúde, inclusive estimulando a elaboração de PTS assentados na lógica interprofissional e colaborativa. Além disso, o contato com ferramentas como genograma, ecomapa e o próprio PTS permitiu vivências antes ausentes na graduação. Assim, esta experiência do PET-Saúde revelou como viável a inclusão em PPC de tais princípios, a fim de promover mudanças na formação dos profissionais e, consequentemente, na produção do cuidado em saúde.

No que tange ao fato de oPET-Saúde ser uma macropolítica disparadora da EIP na formação em saúde, percebe-se seu potencial. Porém, trata-se de uma política pontual que necessita ser incorporada pelas instituições de ensino, nos PPC da área da saúde, para inverter a lógica uniprofissional, curativista e hospitalocêntrica que permeia a sociedade.

O projeto PET-Saúde, em sua totalidade, na UFMS, contou com a participação de cerca de 87 estudantes (bolsistas e voluntários), 20 preceptores e dez tutores que realizaram atendimentos na APS em sete unidades de saúde da capital sul-mato-grossense, contribuindo para a melhoria da qualidade de vida dos usuários do SUS, principalmente no âmbito da promoção de saúde e prevenção de doenças, tornando a experiência da EIP o canal de desenvolvimento da interprofissionalidade e chave para atender às necessidades do setor saúde e do mercado de trabalho.

\section{CONSIDERAÇÕES FINAIS}

A experiência de utilizar a EIP, vivenciar a interprofissionalidade, aprender sobre e com os outros e exercer o cuidado integral e as práticas colaborativas possibilitou mudança na qualidade de vida da paciente, além de proporcionar aprendizados sobre trabalho interprofissional para futuros trabalhadores da saúde ante os desafios do cuidado destinado às condições crônicas.

Por fim, a participação no PET-Saúde permitiu novas vivências na graduação, como o contato com diferentes cursos da saúde, a prática em cenários reais do SUS e a aplicação dos conceitos vistos apenas na teoria, como o atendimento humanizado, o olhar integral e a comunicação com a equipe e a paciente. Apesar da limitação de pacientes alcançados, o programa também oportunizou o reconhecimento dos usuários - a partir do PTS construído e executado - como protagonistas e coprodutores do cuidado em saúde, deslocando o centro 
do cuidado da doença para a pessoa. Reconheceu-se que a formação no SUS e para o SUS não pode prescindir das necessidades de saúde das pessoas, bem como da integralidade da atenção, educando trabalhadores para a organização do trabalho em equipe interprofissional, compromissados com a realidade de saúde da população.

\section{CONTRIBUIÇÃO DOS AUTORES}

Maynara Guaripuna Ferreira, Kathleen Hanna Gonçalves Ferreira, Priscila Cherley de Araújo Ortiz e Adélia Delfina da Motta Silva participaram do desenvolvimento do Projeto Terapêutico Singular que originou o relato. Maynara Guaripuna Ferreira, Cibelle Regina Piani de Souza e Adélia Delfina da Motta Silva participaram da investigação. Maynara Guaripuna Ferreira, Kathleen Hanna Gonçalves Ferreira, Cibelle Regina Piani de Souza e Adélia Delfina da Motta Silva participaram da redação - rascunho original. Rodrigo Guimarães dos Santos Almeida contribuiu com a escrita - revisão e edição. Adélia Delfina da Motta Silva contribuiu com a supervisão, a conceituação, a metodologia e os recursos.

\section{CONFLITO DE INTERESSES}

Declaramos não haver conflito de interesses.

\section{FINANCIAMENTO}

Experiência vinculada ao Edital n 10, 23 de julho 2018, referente à seleção para o Programa de Educação pelo Trabalho para a Saúde - PET-Saúde Interprofissionalidade, projeto 51.

\section{REFERÊNCIASREFERENCES}

1. Brasil. Portaria Interministerial no 1.127, de 4 de agosto de 2015. Institui as diretrizes para a celebração dos Contratos Organizativos de Ação Pública Ensino-Saúde (COAPES), para o fortalecimento da integração entre ensino, serviços e comunidade no âmbito do Sistema Único de Saúde (SUS). Brasília: Ministério da Saúde, Ministério da Educação; 2015 [aceso em $1^{\circ}$ abr 2021]. Disponível em: https://www.uff.br/icvgv/files/2017/06/2.Portaria-Interministerial-N\%c2\%ba-1.127-DE-04-de-Agosto-de-2015.pdf.

2. Brasil. Conselho Nacional de Saúde. Resolução n 569 , de 8 de dezembro de 2017. Brasília: Ministério da Saúde; 2017 [acesso em 31 mar 2021]. Disponível em: http://conselho.saude.gov.br/resolucoes/2017/Reso569.pdf.

3. Brasil. Edital no 10, de 23 de julho 2018 - Seleção para o Programa de Educação pelo Trabalho para a Saúde PET-Saúde/Interprofissionalidade 2018/2019. Diário Oficial da União; 24 jul 2018. Seção 3, p. 78-81.

4. Brasil. Constituição da República Federativa do Brasil: texto constitucional promulgado em 5 de outubro de 1988, compilado até a Emenda Constitucional no 105/2019. Brasília: Senado Federal, Coordenação de Edições Técnicas; 2020, p. 397.

5. Castro FS, Cardoso AM, Penna KGBD. As Diretrizes Curriculares Nacionais dos cursos de graduação da área da saúde abordam as políticas públicas e o sistema único de saúde? Revista Brasileira Militar de Ciências. 2019;5(12):29-34. doi: 10.36414/rbmc.v5i12.11.

6. Batista, NA. Educação interprofissional em saúde: concepções e práticas. Caderno FNEPAS. 2012;2:25-8 [acesso em 14 fev 2020]. Disponível em: https:// edisciplinas.usp.br/pluginfile.php/4298824/mod_resource/content/1/ educacao_interprofissional.pdf.
7. Brasil. Portaria interministerial no 1.802, de 26 de agosto de 2008. Institui - Programa de Educação pelo Trabalho para a Saúde - PET-Saúde. Brasília: Ministério da Saúde, Ministério da Educação; 2008 [acesso em 20 maio 2021]. Disponível em: https://bvsms.saude.gov.br/bvs/saudelegis/ gm/2008/pri1802_26_08_2008.html.

8. Organização Mundial da Saúde. Marco para ação em educação interprofissional e prática colaborativa. Genebra: OMS; 2010 [acesso em 18 fev 2020]. Disponível em: https://www.educacioninterprofesional.org/pt/ marco-para-acao-em-educacao-interprofissional-e-pratica-colaborativa.

9. Ogata MN, Silva JAM, Peduzzi M, Costa MV, Fortuna CM, Feliciano $A B$. Interfaces between permanent education and interprofessional education in health. Rev Esc Enferm USP. 2021;55:e03733. doi: 10.1590/ S1980-220X2020018903733.

10. Olson $\mathrm{R}$, Bialocerkowski A. Interprofessional education in allied health: a systematic review. Med Educ. 2014;48(3):236-46. doi: 10.1111/ medu. 12290

11. Peduzzi M, Norman IJ, Germani ACCG, da Silva JAM, de Souza GC. Interprofessional education: training for healthcare professionals for teamwork focusing on users. Rev Esc Enferm USP. 2013;47(4):977-83. doi 10.1590/S0080-623420130000400029.

12. Brasil. Diretrizes do Nasf: Núcleo de Apoio à Saúde da Família. Brasília: Ministério da Saúde; 2010. (Caderno de Atenção Básica, n²7).

13. Brasil. Núcleo de Apoio à Saúde da Família. Brasília: Ministério da Saúde, 2014. (Cadernos de Atenção Básica, n 39).

14. Hori AA, Nascimento AF. O Projeto Terapêutico Singular e as práticas de saúde mental nos Núcleos de Apoio à Saúde da Família (Nasf) em Guarulhos (SP). Brasil. Ciênc Saúde Colet. 2014;19(8):3561-71. doi: 10.1590/1413-81232014198.11412013.

15. Melo EA, Vianna EC, Pereira LA, organizadores. Caderno do curso Apoio Matricial na Atenção Básica com Ênfase nos Nasf: aperfeiçoamento. 2a ed. Rio de Janeiro: EAD, Ensp, Fiocruz; 2016 [acesso em 14 fev 2020]. Disponível em: https://ead.saude.pe.gov.br/pluginfile.php/3897/mod folder/content/0/caderno-curso-nasf.pdf?forcedownload $=1$.

16. Reis RC, de Souza ARND, Favoreto CAO. Casos complexos na atenção primária à saúde: narrativas de residentes em Medicina de Família e Comunidade. Anais do Congresso Brasileiro de Saúde Coletiva; 2018; Rio de Janeiro. Campinas: Galoá; 2018 [acesso em 31 mar 2021]. Disponível em: https://proceedings. science/saude-coletiva-2018/papers/casos-complexos-na-atencao-primariaa-saude--narrativas-de-residentes-em-medicina-de-familia-e-comunidade.

17. Castro Filho ED, Gusso GDF, Demarzo MMP, Stein A, Schneider JC, Dalla MDB, et al. A especialização em MFC e o desafio da qualificação médica para a Estratégia Saúde da Família: proposta de especialização, em larga escala, via educação à distância. Rev Bras Med Fam Comunidade. 2007;3(9):199-20 [acesso em 12 maio 2021]. Disponível em: https://rbmfc. org.br/rbmfc/article/view/338.

18. Ferreira MG, Ferreira KHG, Ortiz PCA, Silva ADM. PET-Saúde Interprofissionalidade: aprendizagens no uso do projeto terapêutico singular. Saúde e Meio Ambiente: Revista Interdisciplinar. 2020;9(supl1):7980 [acesso em 14 maio 2021]. Disponível em: http://www.periodicos.unc br/index.php/sma/article/view/3403.

19. Chapadeiro CA, Andrade HYSO, de Araújo MRN, organizadores. A família como foco da atenção básica à saúde. Belo Horizonte: Nescon, UFMG; 2012 [acesso em 3 abr 2021]. Disponível em: https://www.nescon.medicina. ufmg.br/biblioteca/imagem/3911.pdf.

20. Dias LC. Abordagem familiar. In: Gusso G, Lopes JMC, Dias LC. Tratado de medicina de família e comunidade: princípios, formação e prática. 2a ed. Porto Alegre: Artmed, 2019.

21. Universidade Federal de Minas Gerais. Projeto Álbum de Família. Belo Horizonte: UFMG; 2011 [acesso em 3 abr 2021]. Disponível em: https:// www.nescon.medicina.ufmg.br/genograma/.

22. Perkins M, Obrecht C, Adams C. Canva: design gráfico para todos. 2012 [acesso em 30 mar 2020]. Disponível em: https://www.canva.com.

23. Libbon R, Triana J, Heru A, Berman E. Family skills for the resident toolbox: the 10-min genogram, ecomap, and prescribing homework Acad Psychiatry. 2019;43(4):435-9 [acesso em 10 abr 2021]. doi: 10.1007/ s40596-019-01054-6. 
24. Brasil. Estratégias para o cuidado da pessoa com doença crônica: diabetes mellitus. Brasília: Ministério da Saúde, 2013. (Cadernos de Atenção Básica, nº 36).

25. Brasil. Clínica ampliada, equipe de referência e projeto terapêutico singular. 2a ed. Brasília: Ministério da Saúde; 2007 [acesso em 15 fev 2020]. Disponível em: http://bvsms.saude.gov.br/bvs/publicacoes/clinica_ampliada_2ed.pdf.

26. Brasil. Manual do pé diabético: estratégias para o cuidado da pessoa com doença crônica. Brasília: Ministério da Saúde, 2016.

27. Mishra SC, Chhatbar KC, Kashikar A, Mehndiratta A. Diabetic foot. BMJ. 2017;16(359):j5064 [acesso em 11 abr 2021]. doi: 10.1136/bmj.j5064.

28. Nather A, Cao S, Chen JLW, Low AY. Prevention of diabetic foot complications. Singapore Med J. 2018;59(6):291-4. doi: 10.11622/smedj.2018069.

29. Mangin $D$, Heath I. Multimorbidade e prevenção quaternária (P4). Rev Bras Med Fam Comunidade. 2015;10(35):1-5 [acesso em 20 maio 2021]. Disponível em: https://rbmfc.org.br/rbmfc/article/view/1069.

30. Ayres JRCM. Sujeito, intersubjetividade e práticas de saúde. Ciênc Saúde Colet. 2001;6(1):63-72. doi: 10.1590/S1413-81232001000100005.

31. Ayres JRCM. Uma concepção hermenêutica de saúde. Physis. 2007;17(1):43-62. doi: 10.1590/S0103-73312007000100004.

32. dos Santos DG, Seixas RAM, Luchesi BM. Avaliação dos impactos promovidos pela oficina de memória "MemorlDADE" para idosos da UnAPI/UFMS. Anais do Integra UFMS; 2020; Campo Grande [acesso em 31 mar 2021]. Disponível em: https://integra.ufms.br/files/2021/01/ Anais_2020_Final.pdf.

33. Healthy Interactions. Programs catalog. Chicago: Healthy Interactions; 2015[acesso em 18 fev 2020]. Disponível em: https://www. healthyinteractions.com/assets/files/HI_Catalog_v01_09_093015.pdf.
34. de Carvalho SL, Ferreira MA, Medeiros JMP, Queiroga ACF, Moreira TR, Silva NFDS. Mapa de conversação: estratégia educativa no cuidado ao idoso com diabetes mellitus. Rev Bras Enferm. 2018;71(supl 2):925-9. doi: 10.1590/0034-7167-2017-0064.

35. dos Anjos KF, Sacramento MS, Ferraz CEO, Vilela ABA, Boery RNSO, Sena ELS. Perspectivas e desafios do núcleo de apoio à saúde da família quanto às práticas em saúde. Saúde Debate. 2013;37(99):672-80. doi: 10.1590/ S0103-11042013000400015.

36. Barr H. Competent to collaborate: towards a competency-based model for interprofessional education. J Interprof Care. 1998;12(2):181-7. doi: doi:10.3109/13561829809014104.

37. Deslandes SF, organizadora. Humanização dos cuidados em saúde: conceitos, dilemas e práticas. Rio de Janeiro: Fiocruz; 2006. doi: 10.7476/9788575413296.

38. Dorigatti AE, Aguilar ML, Madureira RM, da Fonseca FG, Campos RTO, Nascimento JL. Projeto terapêutico singular no âmbito da saúde mental: uma experiência no curso de graduação em medicina. Rev Bras Educ Med. 2014;38(1):113-9. doi: 10.1590/S0100-55022014000100015.

39. Macedo CS, Brandão ICA, Leal JAD, de Oliveira Júnior LG, Cortez RL, Honório MLP, et al. Elaboração de um projeto terapêutico singular para uma família de alto risco em uma unidade básica de saúde, MacaíbaRN: relato de experiência. Revista Eletrônica Extensão \& Sociedade. 2017;6(1):51-66 [acesso em 15 fev 2020]. Disponível em: http://repositorio. ufc.br/bitstream/riufc/35615/1/2017_art_csmacedo.pdf.

40. Mendes KMC, Veloso RV, Teixeira DMT, Banfi MB, da Costa BC, Sousa GV, et al. $O$ uso do Projeto Terapêutico Singular como estratégia para o trabalho interprofissional na atenção pPrimária à saúde: um relato de experiência do Pet Saúde Interprofissionalidade. Enfermagem: Processos, Práticas e Recursos. 2021;(24)236-42. doi: 10.22533/AT.ED.24021240224. 